\title{
LDA Study of Particulate Flow in a Channel with Deformed Surface Locations and with Flow Conditioner
}

\author{
Mehdi Azimian, Hans-Jörg Bart \\ University of Kaiserslautern, Kaiserslautern, Germany \\ Email: bart@mv.uni-kl.de
}

Received 13 November 2015; accepted 24 December 2015; published 28 December 2015

Copyright (C) 2015 by authors and Scientific Research Publishing Inc.

This work is licensed under the Creative Commons Attribution International License (CC BY). http://creativecommons.org/licenses/by/4.0/

(c) (i) Open Access

\section{Abstract}

Hydroabrasion in particulate flows plays an important role in various industrial and natural processes. To predict the effects of particulate flow and the resulting phenomena such as erosion/abrasion in a pipeline, channel or a fitting, it is essential to characterize the effects in a simple standardized geometry. For this purpose, it is vital to initially understand the particulate flow behavior and motion in such geometries. In the present work, two series of experimental works by application of the LDA measurement technique were successfully conducted. First, the particulate flow behavior at downstream of a flow conditioner inside a channel with square cross-section was investigated. Shorter lengths for fully development of velocity profile by using the self-constructed flow conditioner were observed. Moreover, the flow at downstream of the conditioner was modeled with the CFD tool (ANSYS-CFX V. 14.57) and the simulation results were compared and validated by the LDA experimental data. Better agreement between the simulation results and experimental data was observed in the fully developed region. However, there are some deviations due to the actual pressure loss through the experimental loop and the calculated pressure loss value, which includes some assumptions for the loss coefficients. Furthermore, the particulate flow behavior and vortex generation inside the deformed locations of a channel surface were studied in detail. With the help of the Matlab program, it was possible to calculate and visualize the velocity vectors for each measured point inside the channel accurately.

\section{Keywords}

Channel Flow, Flow Conditioner, Particulate Flow, CFD, LDA

\section{Introduction}

Particulate flows are responsible for accelerated erosion/abrasion in pipelines and plant equipment, which can 
significantly reduce the service life and increase the repair and maintenance cost. The existing literature mainly covers Laser/Phase Doppler Anemometry (LDA/PDA) and Particle Image Velocimetry (PIV) measurements of gas-liquid or liquid-liquid multiphase flow systems with relatively few references on solid particles-liquid flows.

Huber and Sommerfeld [1] applied the PDA and a laser light sheet technique to measure the solid particles concentration in a pipe along its cross-section. A very detailed set of experiments was provided by Tsuji and Morikawa [2] for a solid-gas flow in a horizontal pipe using various types of relatively large spherical particles. Ozgoren et al. [3] applied PIV to investigate and compare the flow structures at the downstream region of a cylindrical and spherical obstacle fixed in a channel flow. Kumara et al. [4] compared the PIV and LDA measurement techniques applied to the two-phase oil-water flow in a horizontal pipe. Ristic et al. [5] presented an overview of uncertainty sources in the LDA measurements. They estimated the LDA measuring volume positioning in cylindrical pipe flow due to optical aberrations caused by the pipe wall curvature. Durst et al. [6] applied the LDA method to estimate the local wall shear stress from near-wall mean velocity data in fully developed pipe and channel flows. They validated the Direct Numerical Simulation (DNS) results with the LDA data. Azimian et al. [7] have recently measured the velocity profiles of solid particles and the deviation compared to the liquid phase, velocity fluctuations and the particles size distribution with LDA/PDA technique for solid-liquid flows in a channel with an obstacle positioned perpendicular to the flow direction.

Flow conditioners can accelerate the formation of a fully developed velocity profile and reduce disturbances in the flow [8] [9]. They are necessary, e.g. where a flow measurement tool installed through the flow field. Such conditioners, a number of which are defined in national or international standards, e.g. DIN 1952, allow the reduction of the length of straight pipe required upstream of a flow meter. This obvious advantage must be paid for with an additional pressure loss. Many attempts have been made in optimizing the design of a conditioner such that the desired reduction in straight pipe length is maximized and the inevitable pressure loss $\Delta \mathrm{p}$, minimized. This led to a variety of different approaches in the design of flow conditioners that currently are in practical use.

Schlüter and Merzkirch [8] and Xiong et al. [9] investigated the flow downstream of three different flow conditioners, a tube bundle and two perforated plates, by measuring the time-averaged, axial velocity component with PIV. The conditioners were exposed to the flow disturbed by a $90^{\circ}$ out of plane double-bend. The axial development of the velocity profiles, with and without flow conditioner, was studied. Moreover, the performances of the three devices in conditioning the disturbed flow were compared with each other. Spearman et al. [10] presented the results obtained when locating various designs of perforated plate flow conditioner downstream of two common flow-disturbing installations in turn, as once a twisted $\mathrm{S}$ bend and once a single $90^{\circ}$ bend. The results comprised a series of LDV measurements of velocity and the Root Mean Square (RMS) fluctuation velocity profiles made in two different perpendicular planes downstream of the conditioners. Frattolillo and Massarotti [11] presented a comparison of the performance of different flow conditioners, independently from their effects on particular flow meters. The authors proposed the use of several efficiency parameters to evaluate and compare flatness, uni-directionality and axi-symmetry of the velocity profiles produced downstream of the conditioners. They carried out numerical analysis by using the finite volume technique as well. From the obtained results, it was possible to calculate the efficiency of the conditioners under low-level disturbance conditions, such as those produced by an out of plane double elbow configuration. Manshoor et al. [12] investigated the effects of a fractal flow conditioner for both standard and non-standard flow conditions experimentally and also by using simulations. The experimental results using the fractal flow conditioner showed that the combination of an orifice plate and a fractal flow conditioner was broadly insensitive to upstream disturbances. The simulation results also demonstrated that the device could be used as a part of a flow metering package that would considerably reduce installation lengths. Gordeev et al. [13] focused on a numerical study of the flow conditioner efficiency, in which two different types of flow conditioners were compared. This was done by means of a detailed numerical analysis with respect to specific hydraulic effects in the pipe elbow and the inflow conditioners. The adequateness of three different turbulence models to simulate a flow through a $90^{\circ}$ bend of circular cross-section was investigated.

In this work, the effects of a flow conditioner on the flow behavior (velocity and turbulence profiles) in a channel were experimentally and numerically investigated. The measurement of the velocity and turbulence parameters of the flow took place in a Plexiglas channel with a square cross-section $\left(D_{h}=21 \mathrm{~mm}\right)$ and were carried out with a LDA measurement technique. For the comparison of the velocity profiles, a fitting was used as an aid that adjusted the theoretical fully developed velocity profile for tubes to the experimental data. The de- 
velopment of the velocity profiles and the turbulence intensities was considered as the distance to the conditioner had increased. In addition, the influence of different Reynolds numbers (Re) on the profiles of the velocity and the turbulence intensity in two cases with and without installation of the flow conditioner was investigated. The impact of flow conditioner on the velocity profiles in the channel was investigated numerically with CFD tool as well. The geometry consisted of the upstream with an elbow part, the flow conditioner and the downstream pipe. Velocity profiles at various locations downstream of the flow conditioner were predicted by ANSYSCFX V. 14.57 simulations and were compared and validated by the experimental results obtained from LDA.

For the second series of the LDA experiments, the particulate flow behavior and vortex generation inside the deformed locations of a channel surface were studied in detail. Such deformed surface locations can be found also in industrial components whether purposely or due to erosion. In the case of abrasive solid particles presence in the flow, the particles can rotate in such vortex fields multiple times and therefore can have multi impacts with the surface. The multi impacts per particle per surface position can increase the erosion rate and therefore reduce the service life of the instrument.

\section{Experiments (Flow Conditioner)}

The experimental method of the work is briefly explained here. The experimental apparatus loop is shown in Figure 1. The test section shown in Figure 1 is a channel with deformed surface locations. This test section can be replaced with various test sections e.g. with the channel having a flow conditioner at its upstream. The transparent channel is made of Plexiglas and has a square cross-section. The hydraulic diameter of the channel is 21 $\mathrm{mm}$, the wall's thickness is $4 \mathrm{~mm}$ and the total channel length is $150 \mathrm{~cm}$. For the first series of experiments, the flow conditioner is installed after the elbow part at the upstream of the channel (see Figure 2, left side). The applied flow conditioner is a self-constructed perforated plate, which has 25 evenly arranged holes with a diameter of $2 \mathrm{~mm}$ each. The thickness of the conditioner plate is $10 \mathrm{~mm}$ and it is fixed to the pipe part with screws and seals. The particulate flow is promoted with the help of a centrifugal slurry pump (from Habermann Co.) from the storage tank to the channel in a circuit. The pump has a maximum capacity of $20 \mathrm{~m}^{3} / \mathrm{h}$. The flow rate is measured with a flow meter. A bypass brings a high amount of flow back to the mixing tank to provide a homogeneous flow of water and tracer particles or subsequently the water and sand particles. The LDA laser unit and camera are shown in Figure 1. The laser unit and the detector (camera) are mounted on a traverse system, which is controlled with the software FLOWSIZER 3.0 of TSI. The traverse (by Isel, Germany) provides the measurement of the flow in three coordinate directions with a transformation accuracy of $0.01 \mathrm{~mm}$. The tracer particles are hollow glass particles with the nominal diameter of $8-12 \mu \mathrm{m}$ (from TSI Co.) and have a refractive index of 1.5. The lens which was used has a focal length of $250 \mathrm{~mm}$ for the laser. The light source of the LDA is a water- cooled (up to $4 \mathrm{~W}$ ) Coherent Innova 70C Argon Ion Laser which produces a coherent laser beam.

The measurements in the channel were started when the steady state condition, after some couple of minutes, was achieved in the circuit. The setting of the measurement software was adjusted to measure the velocity at each location point as the average of 20,000 particles passing from the measurement point. The laser was set on $80 \mathrm{~mW}$ and a person could enter the measurement protected area for a short time to adjust the laser and detector

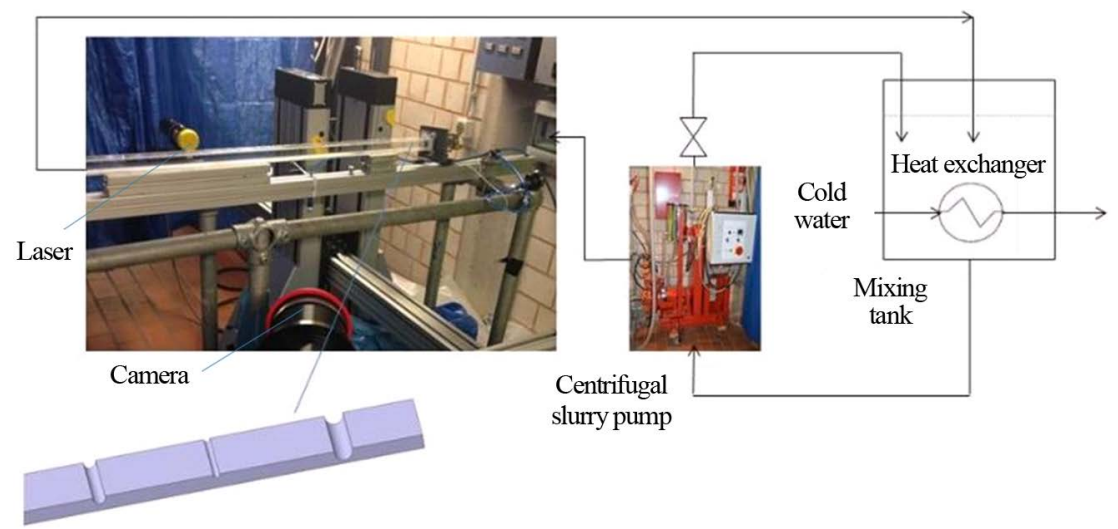

Deformed locations of the channel surface

Figure 1. LDA closed loop experimental apparatus. 


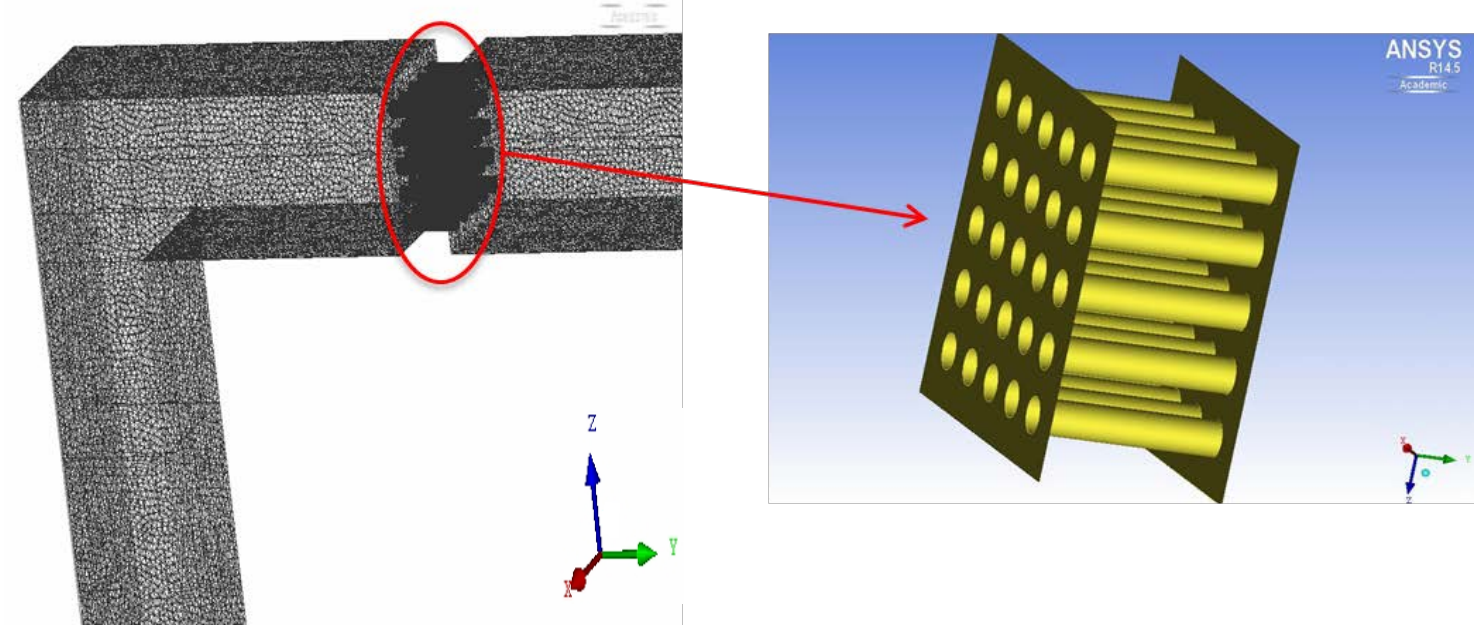

Figure 2. Geometry and mesh generation of the pipe with elbow and flow conditioner.

units. With the help of the software program and preparing the measurement coordinate points in an excel file, it was possible to move the traverse to a desired point by moving along all the three $\mathrm{x}, \mathrm{y}$ and $\mathrm{z}$ directions. The crossing point of two laser beams has to be located in the channel. The detector (camera) could be adjusted using its control window. If the image of the crossing point of two beams was on the label and appeared sharp, the detector was set correctly. This also needs to be accompanied with a good data rate of at least $500-800 \mathrm{~Hz}$ when the laser power is increased to about $500 \mathrm{~mW}$. Also, the efficiency should be at least $10 \%$ - $20 \%$ as a reference value. Afterwards, the velocity profiles were measured each $50 \mathrm{~mm}$ downstream of the flow conditioner for the first series of measurements. The velocity component $\mathrm{V}_{\mathrm{y}}$ was measured, considering the measurement coordinate system shown in Figure 2 (left side).

The LDA experimental results are presented in Figure 3 showing the velocity profiles measured with distance intervals of $50 \mathrm{~mm}$ downstream of the flow conditioner. According to the diagram, the flow was undeveloped at the beginning $(\mathrm{y}=50 \mathrm{~mm}$ ) and became fully developed after about $150 \mathrm{~mm}$ downstream of the flow conditioner. The volumetric feed flow in this case was 900 l/h representing a Re number of 11,869. The velocity magnitude at the upper and bottom walls is obviously zero, however due to the absence of data rate for the software, the velocity magnitudes at these two points are not given as outputs with the software. This is the reason why the velocity profiles in Figure 3 do not start with the zero value on the upper and bottom walls. Having the measured velocity components and velocity fluctuations, the RMS velocity and subsequently the turbulence intensity (Tu) can be calculated and plotted as given by Figure 4 for each measured axial position. The reduction of turbulence intensity by increase in distance of the measured position from the flow conditioner can be observed here clearly. As shown in Figure 4, the turbulence profile becomes also developed and symmetrical by increase the distance from the conditioner. In contrast to the shape of the velocity profile, the turbulence intensity profile has higher values in the vicinity of upper and bottom walls and has a minimum value at the center of the channel.

The velocity profiles were measured by three various volumetric feed flows at different axial locations with and without installation of the flow conditioner. The deviation of the velocity profile for each axial location was calculated in respect to the velocity profile at its former axial location. It is assumed that the velocity profile is fully developed when the deviation becomes less than 3\%. Table 1 presents the velocity profile deviations for the cases with and without flow conditioner by three various feed volumetric flows. For example, the deviation of the velocity profile values from the axial position $50 \mathrm{~mm}$ to the axial position $100 \mathrm{~mm}$ at downstream of the inlet without flow conditioner by $900 \mathrm{l} / \mathrm{h}$ feed flow is $25.9 \%$. It is observed that the flow becomes fully developed at a distance of $200 \mathrm{~mm}$ after the inlet part of the channel in the case without the flow conditioner. By applying the flow conditioner, it was observed that the length for full development of flow decreases. As it is demonstrated in Table 1, this length reduces to $150 \mathrm{~mm}$ with feed flow of $900 \mathrm{l} / \mathrm{h}$. By increasing the volumetric flow to 1400 and $1900 \mathrm{l} / \mathrm{h}$, the length was reduced further to $100 \mathrm{~mm}$. 


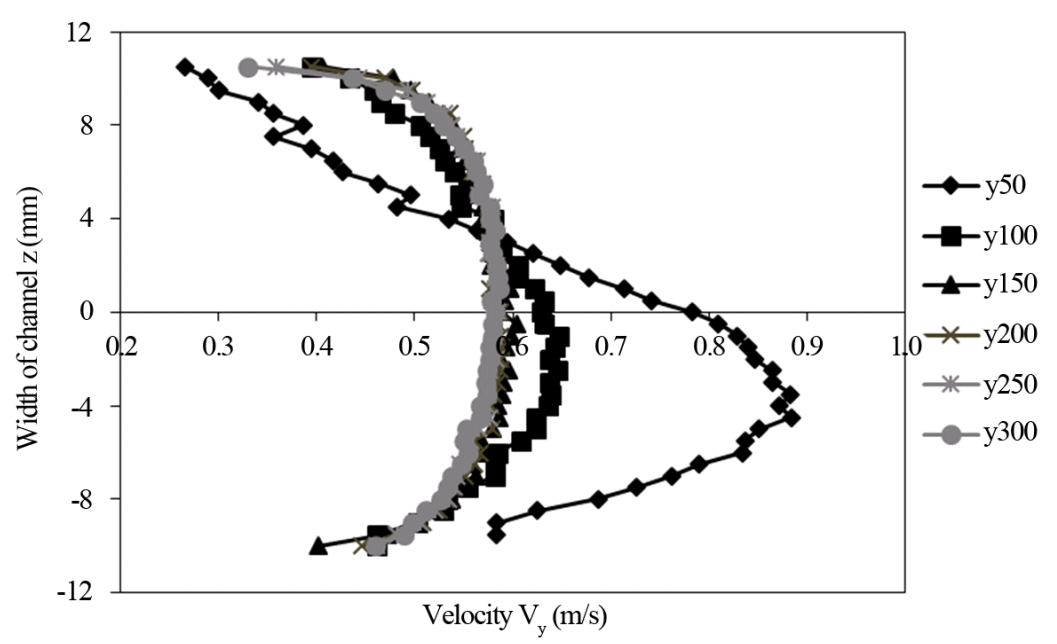

Figure 3. LDA measured velocity profiles downstream of flow conditioner with $\dot{\mathbf{V}}=900 \mathrm{l} / \mathrm{h}$.

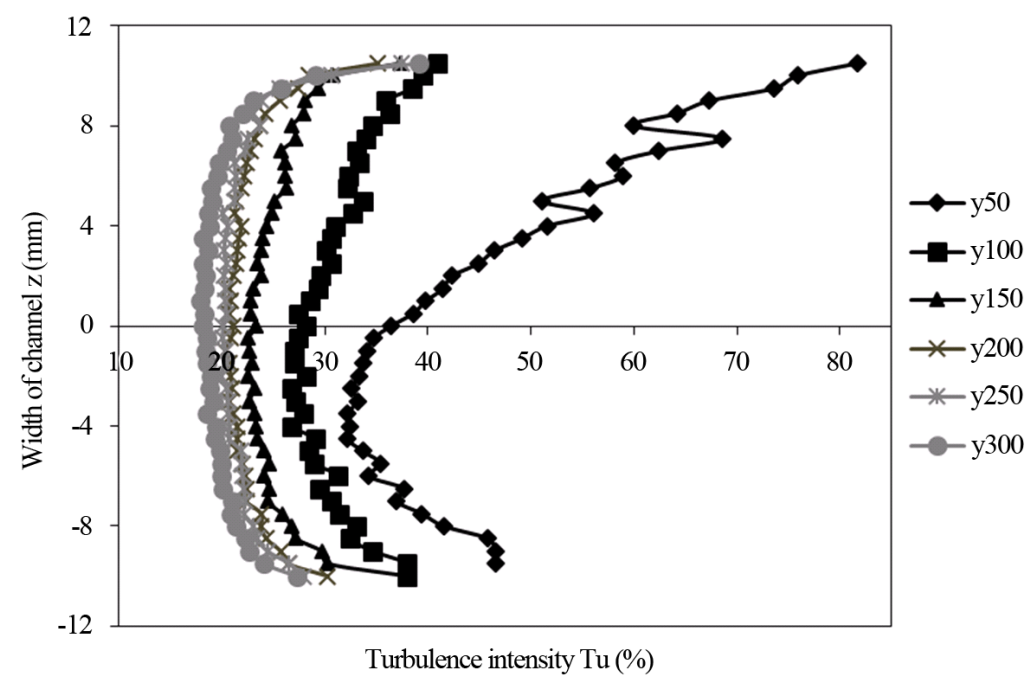

Figure 4. LDA measured turbulence intensity profiles downstream of flow conditioner with $\dot{\mathbf{V}}=900 \mathrm{l} / \mathrm{h}$.

Table 1. Velocity profiles deviation for the cases with and without flow conditioner.

\begin{tabular}{ccccccc}
\hline \multirow{2}{*}{$\begin{array}{c}\text { Downstream } \\
\text { velocity profile } \\
\text { deviation between axial } \\
\text { positions }\end{array}$} & \multicolumn{3}{c}{ Without flow conditioner } & \multicolumn{3}{c}{ With flow conditioner } \\
\cline { 2 - 7 } & $900 \mathrm{l} / \mathrm{h}$ & $1400 \mathrm{l} / \mathrm{h}$ & $1900 \mathrm{l} / \mathrm{h}$ & $900 \mathrm{l} / \mathrm{h}$ & $1400 \mathrm{l} / \mathrm{h}$ & $1900 \mathrm{l} / \mathrm{h}$ \\
\cline { 2 - 7 } & $\%$ & $\%$ & $\%$ & $\%$ & $\%$ & $\%$ \\
\hline $50 \mathrm{~mm}-100 \mathrm{~mm}$ & 25.90 & 26.10 & 23.23 & 24.59 & 21.96 & 20.17 \\
$100 \mathrm{~mm}-150 \mathrm{~mm}$ & 8.69 & 7.77 & 6.38 & 4.91 & 2.62 & 2.43 \\
$150 \mathrm{~mm}-200 \mathrm{~mm}$ & 4.07 & 4.57 & 4.41 & 1.25 & 2.84 & 3.28 \\
$200 \mathrm{~mm}-250 \mathrm{~mm}$ & 2.48 & 2.07 & 2.00 & 0.99 & 1.62 & 2.45 \\
$250 \mathrm{~mm}-300 \mathrm{~mm}$ & 1.09 & 1.22 & 0.85 & 0.87 & 0.94 & 1.52 \\
$300 \mathrm{~mm}-350 \mathrm{~mm}$ & 0.99 & 0.69 & 0.92 & & 0.97 & 1.05 \\
$350 \mathrm{~mm}-400 \mathrm{~mm}$ & 1.13 & 0.79 & 0.79 & & & 1.06 \\
\hline
\end{tabular}




\section{CFD Modeling}

The geometries of the square cross-sectional channel, the elbow and the flow conditioner were generated with the ANSYS-ICEM tool based on the exact dimensions in the experiments. As depicted in Figure 2, the flow conditioner with the thickness of $10 \mathrm{~mm}$ is located at a distance of $50 \mathrm{~mm}$ to the elbow part. The geometry of flow conditioner (see Figure 2, right side) was generated separately and later was merged to the other two geometries of upstream and downstream parts. After merging the geometry parts together, a tetrahedral mesh was generated with the "standard tetra mesher" option. The standard tetra mesher requires a surface mesh of tri or quad elements as input, and then provides a number of options to control the resulting tetrahedral mesh. The generation of tetrahedral mesh was necessary to connect the fine mesh of the round conditioner holes to the mesh of downstream and upstream parts without any error (see Figure 2, left side). The velocity profiles were predicted with the help of CFD tool at downstream of the flow conditioner at distances with $50 \mathrm{~mm}$ intervals. CFD results were compared with the LDA measurement data at each measured location. As examples, the results from LDA and CFD with the volumetric feed flow of $900 \mathrm{l} / \mathrm{h}$ at distances of 50 and $150 \mathrm{~mm}$ downstream of the flow conditioner are shown in Figure 5 and Figure 6, respectively. As depicted, the velocity profile at $50 \mathrm{~mm}$ downstream of the flow conditioner is disturbed and gradually develops as the distance to the flow conditioner increases. Based on the derived results presented in Table 1, the velocity profile at the distance of $150 \mathrm{~mm}$ downstream of the flow conditioner can be considered as fully developed. Overall, a good qualitative agreement between the CFD results and the LDA experimental data was achieved. However, a better agreement between the simulation results and the experimental data was observed in the fully developed region. The deviations are mainly due to the actual pressure loss through the experimental loop and the calculated pressure loss value which includes some assumptions for the loss coefficients of the components (e.g. loss coefficients of the used pipe, elbow, flow conditioner, etc.).

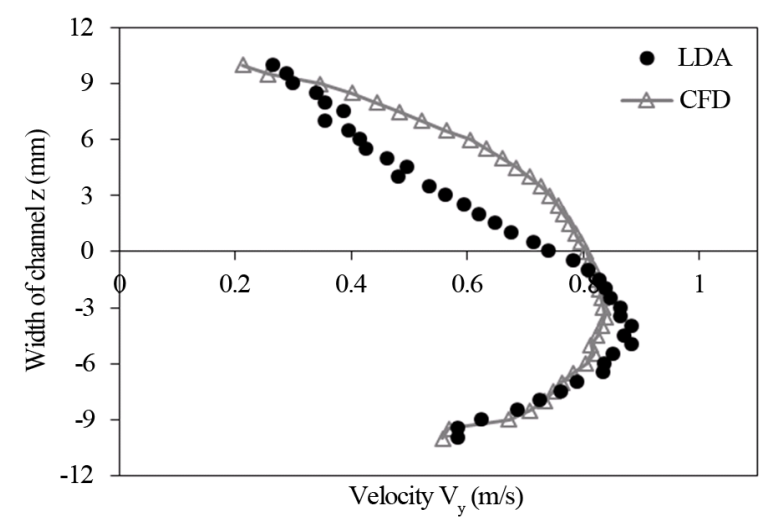

Figure 5. LDA/CFD comparison with the flow conditioner at $\mathrm{y}=+50 \mathrm{~mm}$.

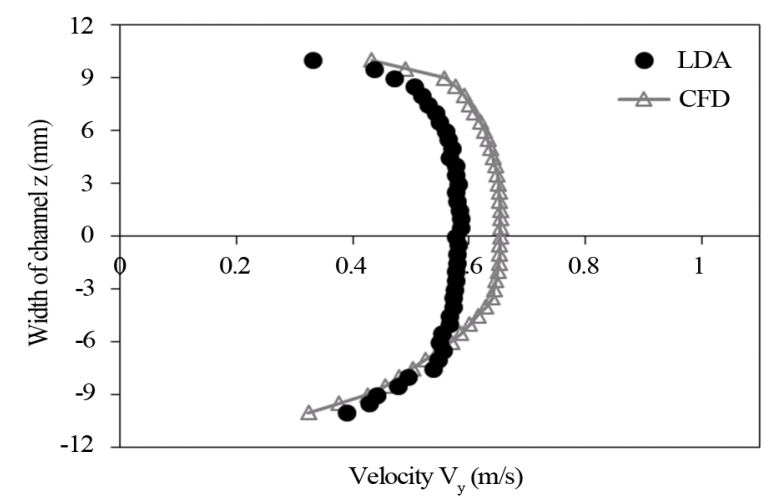

Figure 6. LDA/CFD comparison with the flow conditioner at $\mathrm{y}=+150 \mathrm{~mm}$. 


\section{Experiments (Deformed Surface Channel)}

The second series of measurements were carried out in a channel with deformed surface locations. The channel made from Plexiglas has a length of $1200 \mathrm{~mm}$ and a square cross-section with the inner width of $28 \mathrm{~mm}\left(\mathrm{D}_{\mathrm{h}}\right)$. The three deformed locations are half cylindrical shape holes drilled through the width of the channel (perpendicular to the flow direction) with diameters of $20 \mathrm{~mm}, 10 \mathrm{~mm}$ and $30 \mathrm{~mm}$, respectively. Figure 7 shows the channel with the deformed locations, measurement locations (dotted vertical lines inside the channel), flow direction and measurement coordinate. The motivation of this study was to predict the behavior of particulate flow through a deformed surface of a channel and to investigate the effects on the erosion behavior in such locations. As an illustrating example, a deformed surface of a river bed is shown in Figure 8 and the vortex generation is defined clearly in such location [14]. This phenomenon can occur also in industrially applied components whether purposely or due to erosion. In the case of abrasive solid particles presence in the flow, the particles can rotate in such vortex fields multiple times and therefore can have multi impacts with the surface. The multiple impacts per particle per surface position can increase the erosion rate and therefore reduce the service life of the instrument.

First of all, the velocity profiles were measured at defined positions shown in Figure 7 with the volumetric feed flow of $2600 \mathrm{l} / \mathrm{h}$ and by addition of tracer particles into the water flow. The distances between the holes in the channel are enough long to guaranty the regeneration of the fully developed velocity profile at downstream of each hole. Positions 2, 4 and 6 are located exactly at the center of each hole and the velocity component $\left(\mathrm{V}_{\mathrm{y}}\right)$ was measured along the z-coordinate direction with intervals of $1 \mathrm{~mm}$ from bottom of the hole to the top point of the upper wall as depicted in Figure 7. The velocity profiles were also measured at two positions at upstream and downstream of each hole as shown in Figure 7. As an example, the velocity profiles measured at positions 1, 2 and 3 are depicted in Figure 9. As shown, the velocity profiles at positions 1 and 3 represent relatively the fully developed velocity profile in the channel. Velocity profile at position 2 is also shown here. The two gray dashed lines in Figure 9 represent the bottom and upper surface of the channel. Therefore, the measured points under the bottom dashed line represent the measured points inside the cylindrical hole. The negative values of the measured points at the lower part of the hole, demonstrate that the particles are moving in opposite direction to the main flow direction and therefore, a vortex is generated inside the hole. The velocity profiles at positions 2, 4 and 6 (deformed surface locations) are compared with each other in Figure 10. As shown, by increase in the size of the hole, the negative values of the velocity at lower parts of the hole increase and therefore, faster and bigger vortex fields were generated. The velocity profile of position 4 (containing smallest hole with the diameter of $10 \mathrm{~mm}$ ) shown in Figure 10, illustrates that there are no measured points inside the hole with negative values and therefore, no vortex was generated at this position. This was also approved with the high-speed camera measurements that the flow passes above the hole without any formation of vortex, under the

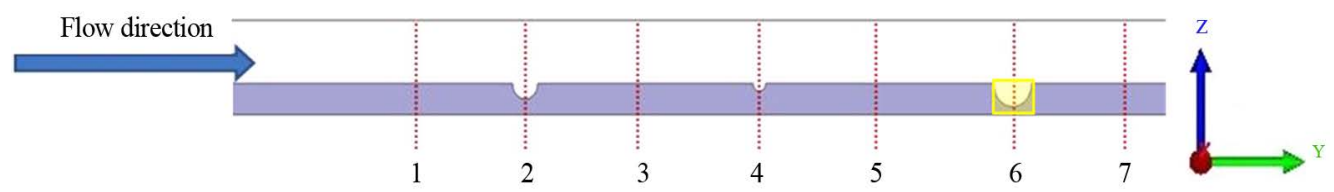

Figure 7. Measurement locations in a channel with deformed surface locations.

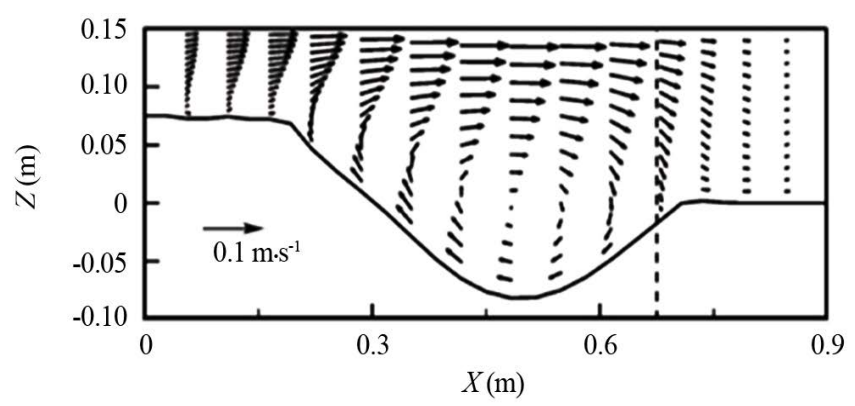

Figure 8. Vortex generation in a deformed location of a river bed surface [14]. 


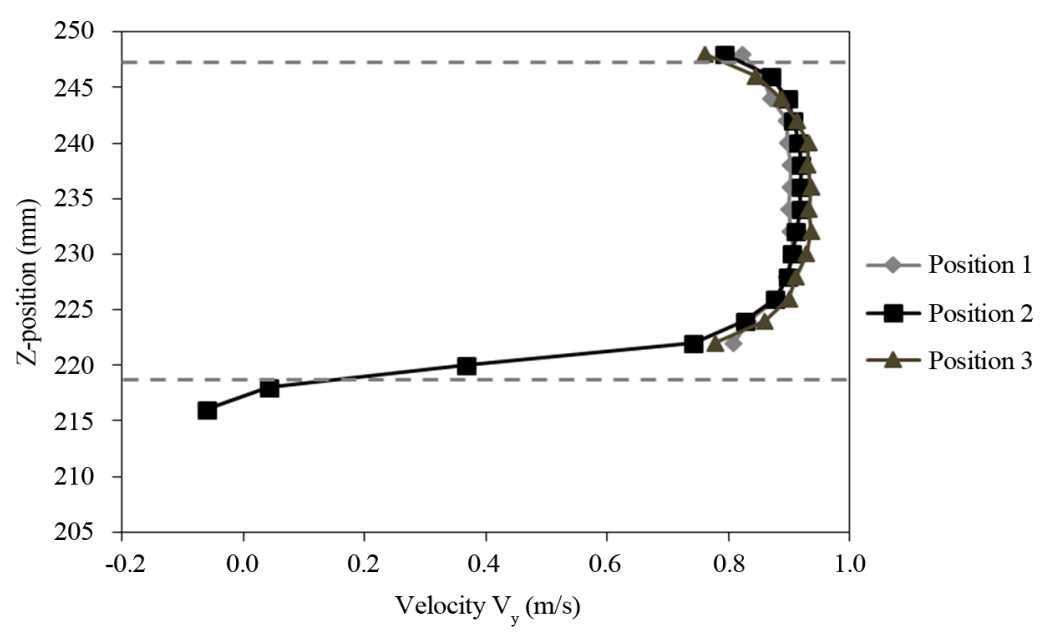

Figure 9. Velocity profiles of the tracer particles at positions 1, 2 and 3.

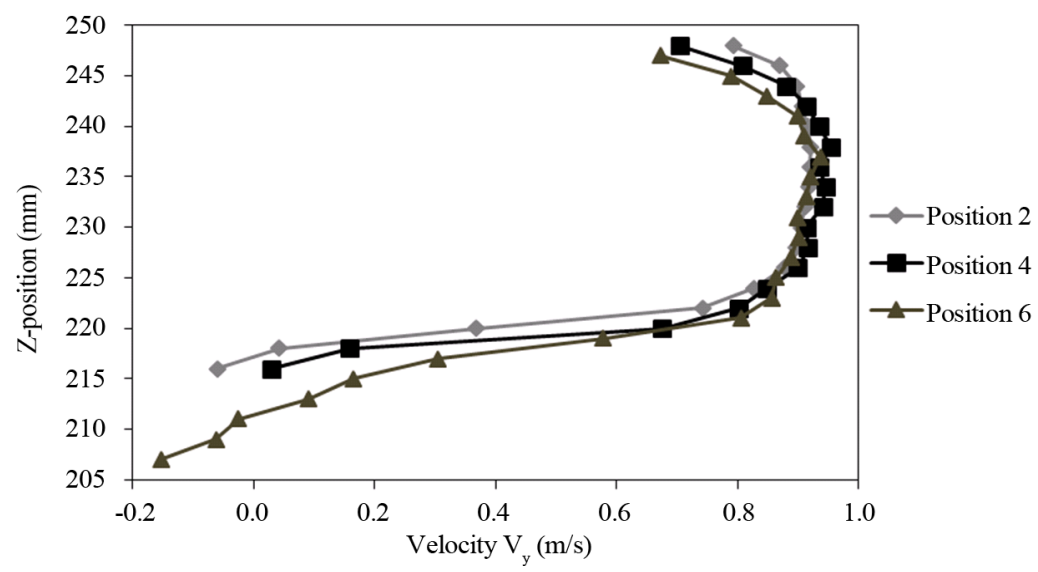

Figure 10. Velocity profiles of the tracer particles at positions 2, 4 and 6.

applied volumetric feed flow conditions for the current study.

With the help of the traverse system, the 3D velocity profile at any position can be also measured. In Figure 11, the 3D velocity profile (velocity in y direction) was measured inside the third hole (the area highlighted in Figure 7), by transforming the traverse system in both $\mathrm{z}$ and $\mathrm{x}$ coordinate directions. Positive velocity values at upper part of the hole and negative velocity values at lower part of the hole express the existence of the vortex field inside the hole. The vortex generation and the multiple impacts of particles with surface inside the hole were observed with the help of high-speed camera as well. From the high-speed camera measurements, it was observed that the sand particles inside the hole can rotate multiple times by the vortex field and have randomly multi impacts with the surface of the hole. The arrow shown in Figure 12 defines the vortex motion inside the hole. The particulate flow direction in the channel shown in Figure 12 is from left to right. The sand concentration for the tests was $0.1 \mathrm{wt} . \%$.

Moreover, the velocity was measured in two directions of $\mathrm{y}$ and $\mathrm{z}$ inside the third hole (the area highlighted in Figure 7). For this purpose, the LDA with two lasers (green and blue) for the main flow direction and perpendicular direction to the flow was applied. In this way, the velocity components of each particle in $\mathrm{y}$ and $\mathrm{z}$ directions were measured. With these two measured data, the resultant value of the velocity vector and the angle of the vector were calculated using the Pythagorean theory equation. The location of each particle in coordinate system was also known. Having all these information, the measured velocity vectors were derived with the Matlab program. Figure 13 shows the measured velocity vectors of 12 coordinate points inside the third hole in flow direction (the area highlighted in Figure 7). The measured $V_{y}$ and $V_{z}$ for each point is the averaged value of 5000 measurements of the passing particles from that point. The size of each array shows relatively the 


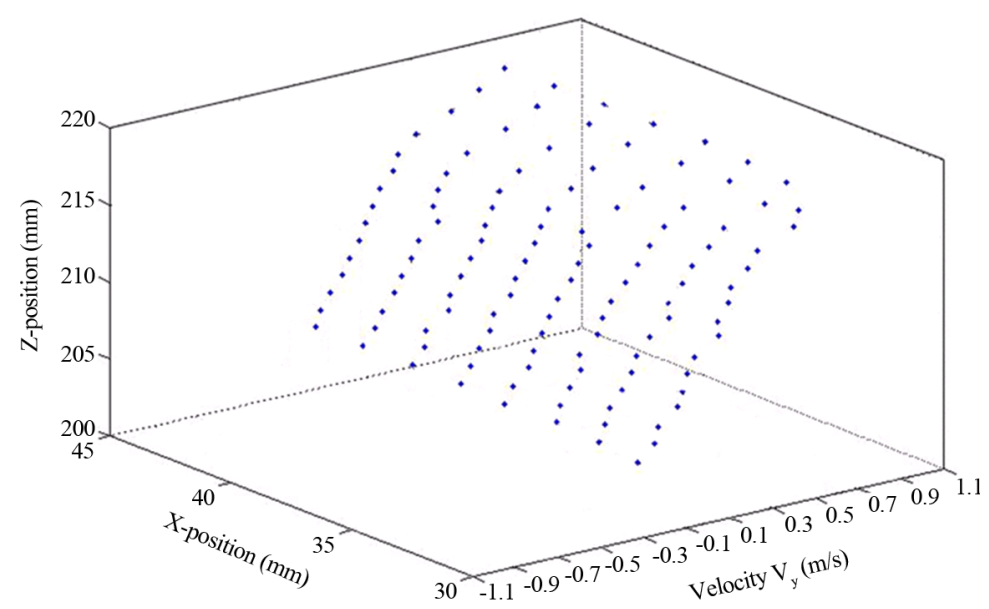

Figure 11. 3D velocity profile inside the third hole (the area highlighted in Figure 7).

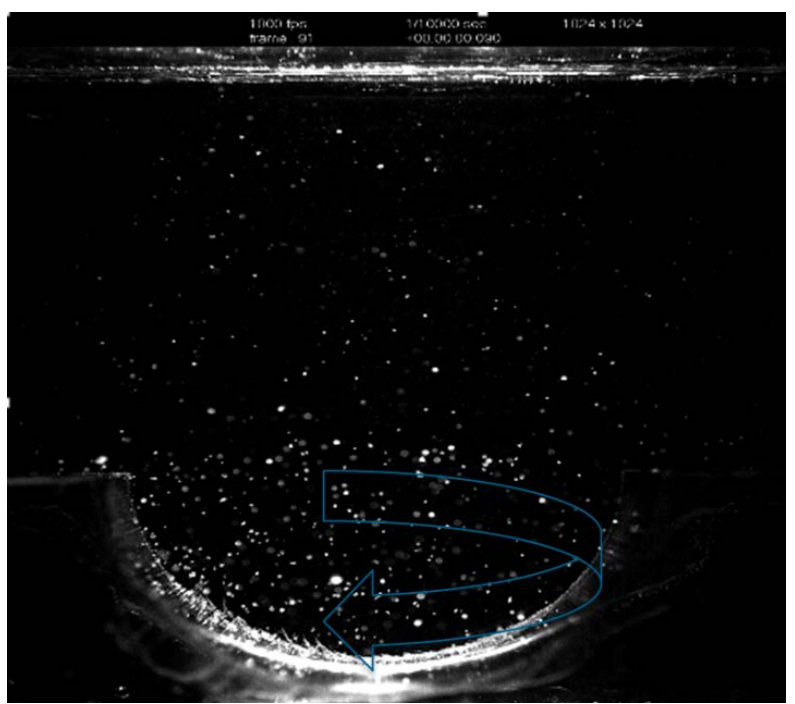

Figure 12. Formation of vortex field, imaged with the high-speed camera.

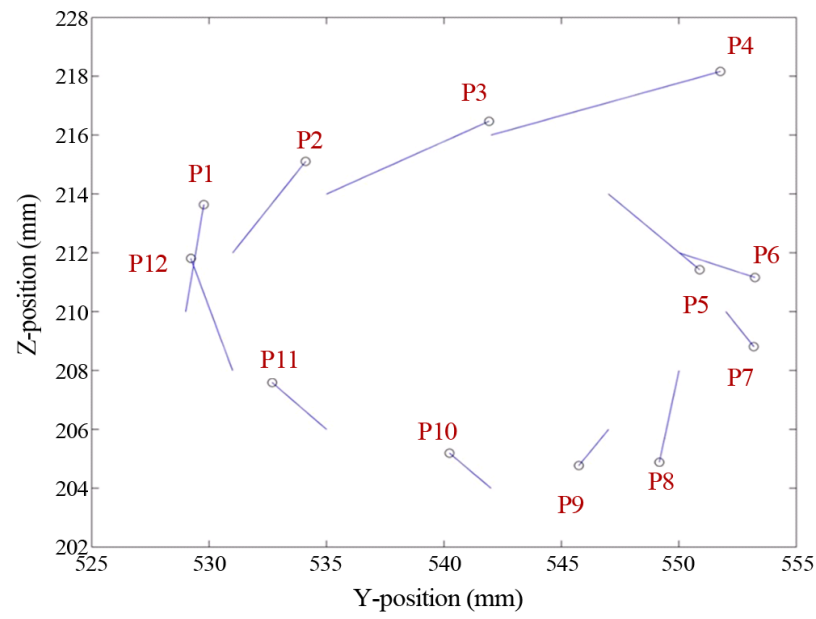

Figure 13. Measured velocity vectors inside the third hole $(\dot{\mathbf{V}}=36001 / \mathrm{h})$. 
magnitude of the velocity vector in comparison with the maximum velocity. The velocities at upper part of the hole are bigger and are in the direction of the main flow in channel (from left to right). However, the velocities at lower part of the hole are relatively smaller and are in opposite direction to the main flow. The unfilled circle at the end of each arrow shows the direction of each moving particle. The advantage of the LDA measurement technique compared to the PIV technique is therefore, the ability to measure the exact flow characteristics at each desired location point.

\section{Conclusion}

Two series of experimental works by application of the LDA measurement technique were carried out. First, the particulate flow behavior at downstream of a flow conditioner inside a channel with a square cross-section was investigated. Shorter lengths for development of velocity profile by using the flow conditioner were observed. Moreover, the flow at downstream of the flow conditioner was modeled with the CFD tool (ANSYS-CFX V. 14.57) and the simulation results were compared and validated by the LDA experimental data. Overall, a good qualitative agreement between the CFD results and LDA experimental data was achieved. However, a better agreement between the simulation results and the experimental data was observed in the fully developed region. The deviations are mainly due to the actual pressure loss through the experimental loop and the calculated pressure loss value, which includes some assumptions for the loss coefficients of the components. Furthermore, the particulate flow behavior and vortex generation inside the deformed locations of a channel surface were studied in detail. With the help of the Matlab program, it was possible to calculate the velocity vector for each measured point inside the channel precisely. In the case of abrasive solid particles presence in the flow, the particles can rotate in such vortex fields multiple times and therefore can have multi impacts with the surface. The multi impacts per particle per surface position can increase the erosion rate and therefore reduce the service life of the instrument.

\section{Acknowledgements}

The authors would like to thank “Stiftung Rheinland-Pfalz für Innovation, Mainz, Germany” for financial support.

\section{References}

[1] Huber, N. and Sommerfeld, M. (1994) Characterization of the Cross-Sectional Particle Concentration Distribution in Pneumatic Conveying Systems. Powder Technology, 79, 191-210. http://dx.doi.org/10.1016/0032-5910(94)02823-0

[2] Tsuji, Y. and Morikawa, Y. (1982) LDV Measurements of an Air-Solid Two-Phase Flow in a Horizontal Pipe. Journal of Fluid Mechanics, 120, 385-409. http://dx.doi.org/10.1017/S002211208200281X

[3] Ozgoren, M., Pinar, E., Sahin, B. and Akilli, H. (2011) Comparison of Flow Structures in the Downstream Region of a Cylinder and Sphere. International Journal of Heat and Fluid Flow, 32, 1138-1146. http://dx.doi.org/10.1016/j.ijheatfluidflow.2011.08.003

[4] Kumara, W.A.S., Elseth, G., Halvorsen, B.M. and Melaaen, M.C. (2010) Comparison of Particle Image Velocimetry and Laser Doppler Anemometry Measurement Methods Applied to the Oil-Water Flow in Horizontal Pipe. Flow Measurement and Instrumentation, 21, 105-117. http://dx.doi.org/10.1016/j.flowmeasinst.2010.01.005

[5] Ristic, S., Ilic, J., Cantrak, D., Ristic, O. and Jankovic, N. (2012) Estimation of Laser-Doppler Anemometry Measuring Volume Displacement in Cylindrical Pipe Flow. Thermal Science, 16, 1027-1042. http://dx.doi.org/10.2298/TSCI1204027R

[6] Durst, F., Kikura, H., Lekakis, I., Jovanović, J. and Ye, Q. (1996) Wall Shear Stress Determination from Near-Wall Mean Velocity Data in Turbulent Pipe and Channel Flows. Experiments in Fluids, 20, 417-428. http://dx.doi.org/10.1007/BF00189380

[7] Azimian, M., Lichti, M. and Bart, H.-J. (2014) Investigation of Particulate Flow in a Channel by Application of CFD, DEM and LDA/PDA. The Open Chemical Engineering Journal, 8, 1-11. http://dx.doi.org/10.2174/1874123101408010001

[8] Schlüter, T. and Merzkirch, W. (1996) PIV Measurements of the Time-Averaged Flow Velocity Downstream of Flow Conditioners in a Pipeline. Flow Measurement and Instrumentation, 7, 173-179. http://dx.doi.org/10.1016/S0955-5986(96)00016-7

[9] Xiong, W., Kalkühler, K. and Merzkirch, W. (2003) Velocity and Turbulence Measurements Downstream of Flow 
Conditioners. Flow Measurement and Instrumentation, 14, 249-260.

http://dx.doi.org/10.1016/S0955-5986(03)00031-1

[10] Spearman, E., Sattary, J. and Reader-Harris, M. (1996) Comparison of Velocity and Turbulence Profiles Downstream of Perforated Plate Flow Conditioners. Flow Measurement and Instrumentation, 7, 181-199.

http://dx.doi.org/10.1016/S0955-5986(96)00013-1

[11] Frattolillo, A. and Massarotti, N. (2002) Flow Conditioners Efficiency a Comparison Based on Numerical Approach. Flow Measurement and Instrumentation, 13, 1-11. http://dx.doi.org/10.1016/S0955-5986(02)00017-1

[12] Manshoor, B., Nicolleau, F. and Beck, S. (2011) The Fractal Flow Conditioner for Orifice Plate Flow Meters. Flow Measurement and Instrumentation, 22, 208-214. http://dx.doi.org/10.1016/j.flowmeasinst.2011.02.003

[13] Gordeev, S., Gröschel, F., Heinzel, V., Hering, W. and Stieglitz, R. (2014) Numerical Study of the Flow Conditioner for the IFMIF Liquid Lithium Target. Fusion Engineering and Design, 89, 1751-1757. http://dx.doi.org/10.1016/j.fusengdes.2013.12.010

[14] Liu, C. and Shen, Y.M. (2009) A Three-Dimensional Solid-Liquid Two-Phase Turbulence Model with the Effect of Vegetation in Non-Orthogonal Curvilinear Coordinates. Science in China Series G: Physics, Mechanics and Astronomy, 52, 1062-1073. http://dx.doi.org/10.1007/s11433-009-0136-8 